\section{Dark clouds of contrast}

\author{
M. Boulaksil (D) J. E. M. Mellema • T. J. F. ten Cate
}

Published online: 14 May 2019

(C) The Author(s) 2019

\section{Answer}

Notably, the two-vessel disease consisted of an obtuse marginal branch and two lesions in the left anterior descending (LAD) artery (Fig. la and online video). We decided on percutaneous coronary intervention (PCI) of the LAD. However, the distal lesion was partially localised in an intramural segment of the LAD (so called 'bridging'). After direct stenting of the proximal LAD lesion, the distal lesion was predilated and a drug-eluting stent was implanted. Thereafter, 'chimneys of contrast' were perceived running into the myocardium and pericardium (arrow head in Fig. la and online video). These chimneys are extravasations of contrast caused by coronary perforation.

Coronary perforation in PCI occurs in $0.2-0.3 \%$ of cases, depending on PCI complexity, and is associated with increased mortality risk $[1,2]$. For grading of coronary perforation, the Ellis classification is most commonly used [3]. Implantation of a covered stent is one of the therapeutic options, though with an increased risk of in-stent thrombosis and restenosis [2].

In our patient, a covered stent was subsequently implanted to seal the perforation (Fig. 1b). Although the clinical course was complicated by recurrent

\section{Electronic supplementary material The online version of this article (https://doi.org/10.1007/s12471-019-1258-x) contains supplementary material, which is available to authorized users.}

\author{
M. Boulaksil ( $\square)$ · T. J. F. ten Cate \\ Department of Cardiology, Radboud University Medical \\ Center, Nijmegen, The Netherlands \\ m.boulaksil@gmail.com
}

\section{J. E. M. Mellema}

Department of Cardiology, Slingeland Hospital,

Doetinchem, The Netherlands episodes of mild pericarditis, our patient could be discharged five days after the PCI. This case emphasises that stenting of intra-mural coronary arteries, even when partially intramural, should be discouraged.

Conflict of interest M. Boulaksil, J.E.M. Mellema and T.J.F. ten Cate declare that they have no competing interests.

Open Access This article is distributed under the terms of the Creative Commons Attribution 4.0 International License (http://creativecommons.org/licenses/by/4.0/), which permits unrestricted use, distribution, and reproduction in any medium, provided you give appropriate credit to the original author(s) and the source, provide a link to the Creative Commons license, and indicate if changes were made.

\section{References}

1. British Cardiovascular Intervention Society, National Institute for Cardiovascular Outcomes Research, Kinnaird T, Kwok CS, Kontopantelis E, British Cardiovascular Intervention Society and the National Institute for Cardiovascular Outcomes Research. Incidence, determinants, and outcomes of coronary perforation during percutaneous coronary intervention in the United Kingdom between 2006 and 2013: an analysis of 527121 cases from the British Cardiovascular Intervention Society Database. Circ Cardiovasc Interv. 2016;9:e3449.

2. Guttmann OP, Jones DA, Gulati A, et al. Prevalence and outcomes of coronary artery perforation during percutaneous coronary intervention. EuroIntervention. 2017;13:e595-e601.

3. Ellis SG, Ajluni S, Arnold AZ, et al. Increased coronary perforation in the new device era. Incidence, classification, management, and outcome. Circulation. 1994;90:2725-30. 

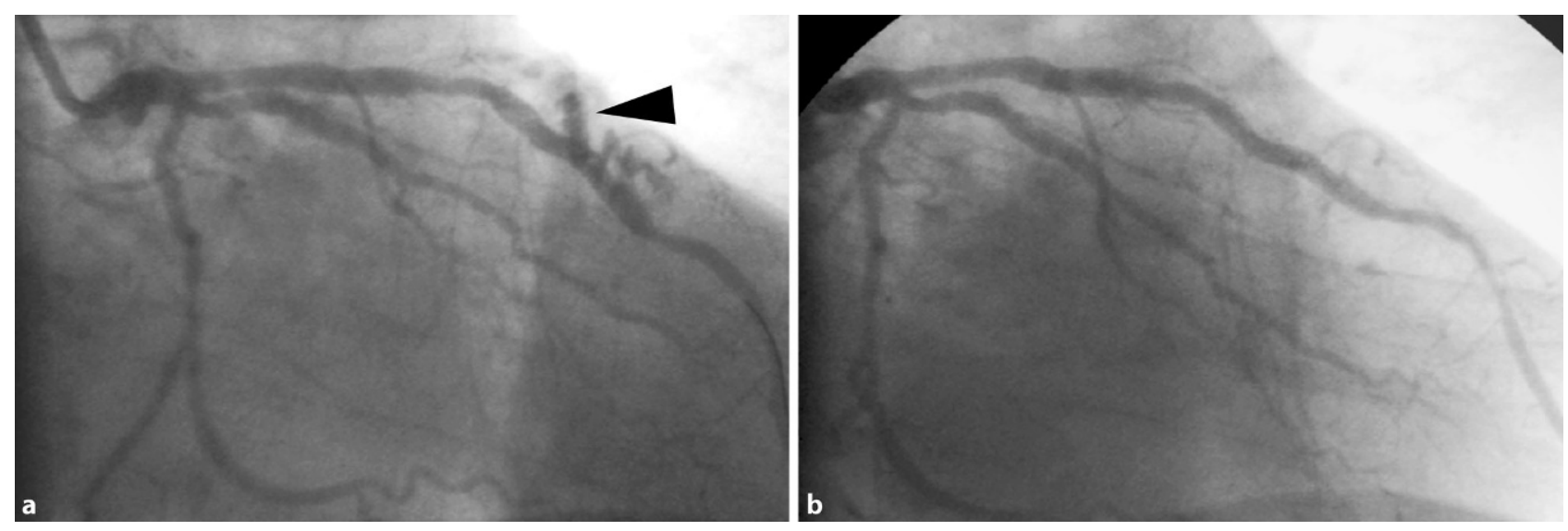

Fig. 1 a Still image of right anterior oblique (RAO) view of coronary angiogram of our patient. The arrow head shows a chimney of contrast indicative of contrast extravasation

caused by coronary perforation. See also the online video (online supplement). b Still image (RAO view) showing the final result after covered stent implantation 